\title{
Inhibition of pupillary orienting reflex by heteromodal novelty*
}

\author{
RICK M. GARDNER, SUCHOON S. MO, and RICHARD KRINSKY \\ Southern Colorado State College, Pubelo, Colorado
}

\begin{abstract}
When recognition memory is well formed, pupillary orienting reflex to novel auditory stimulus items is inhibited. Instead, pupillary dilation occurs to familiar stimulus items provided the task is relatively difficult. The relation between orienting reflex and recognition memory is interpreted in terms of negative induction arising from the direction of the reflex in the context of Pavlov's theory.
\end{abstract}

Sokolov's theory of the orienting reflex (Sokolov, 1963) does not specify the role of the task requirement with respect to the relationship between the orienting and the conditioned reflex, probably because an important implication arising from Pavlov's original theory-the implication that the orienting and the conditioned reflex are distinguishable from each other in terms of their directionality-is not seriously taken into consideration. According to Pavlov's theory (Pavlov, 1927), the mechanism of the orienting reflex is regarded as identical to the mechanism of external inhibition (Pp. 12-13, 44), and it is assumed that there is a relationship of reciprocal induction between these mechanisms (Pp. 382). This assumption implies that directions of the orienting and the conditioned reflex are externalization and internalization, respectively. The former is associated with collection of information; the latter is associated with storage of information. If the task requires that direction of the reflex is relevant, then the effect of such a task requirement should manifest itself through reciprocal induction.

Specifically, assume that the task requirement is that of forming recognition memory and that pupillary dilation to a novel stimulus is an orienting reflex. Since directionality of memory is toward internalization, the effectiveness of the task requirement in maintaining reflex directionality toward internalization should manifest itself in negative induction of the orienting reflex. That is, the novel stimulus should elicit pupillary constriction or, at least, inhibition of dilation, not pupillary dilation. This prediction is quite opposite to what would be predicted on the basis of Sokolov's theory.

This broad interpretation of Pavlov's original theory in terms of reflex directionality and task requirement has been supported by a previous study (Gardner, Mo, \& Borrego, 1974) which showed elicitation of pupillary constriction, not dilation, to a novel stimulus when the

*This study was supported in part by funds from the Faculty Research and Professional Growth Committee, Southern Colorado State College. The second author is responsible for the Colorado State College. The second author is responsible for the
broad interpretation of Pavlov's theory with respect to the orienting reflex. task required formation of recognition memory. However, the study used visual stimuli and the possibility that observed pupillary reaction was caused by factors unique to visual modality was not ruled out. The present study attempts to demonstrate pupillary constriction to a novel auditory stimulus.

\section{EXPERIMENT I}

\section{Method}

Four undergraduate volunteers served as Ss. Each S was presented a list of 10-high-frequency words for $1.5 \mathrm{sec}$ each, with no spacing, on a Stowe memory drum. Word frequency was determined through use of items ranked 25 to 50 on the Thorndike and Lorge scale of frequency (Thorndike \& Lorge, 1944). Immediately after each presentation of the inspection list, each $\mathrm{S}$ was tested for recognition memory by being presented with a mimeographed sheet of 10 items previously presented, together with 10 randomly intermixed new filler items of similar frequency, with the instruction to designate those items previously presented. This procedure was repeated until a criterion of two perfect recognition trials was reached.

After the criterion was met, each $\mathrm{S}$ was seated approximately $2 \mathrm{~m}$ before a Craig video camera. S's head rested in a frame that deflected an image of his right pupil via a mirror into the camera with the magnification factor set at 9 . Test items were verbal tape recordings consisting of 10 old items and 10 randomly intermixed new filler items of similar word-frequency level. The test items were presented at a rate of one item per $10 \mathrm{sec}$ biaurally through earphones. The sound-pressure level was maintained at 76 to $80-\mathrm{dB}$ range. Ss were instructed to report verbally at each presentation whether the item heard was an old or a new item.

\section{Results}

The videotaped record of S's pupil was displayed on a TV monitor, and pupil diameter was measured. One measurement per second was made. Mean pupil diameter was calculated for each learned (old) and unlearned (new) item. In calculating the means, the data from the first item presented were deleted because of disproportionately large pupillary dilation.

Figure 1 shows changes in mean pupil diameter for old and new items throughout the 10-sec interval. Item presentation occurred at $0 \mathrm{sec}$. For each $\mathrm{S}$, the mean latency between onset of the item and the verbal report 


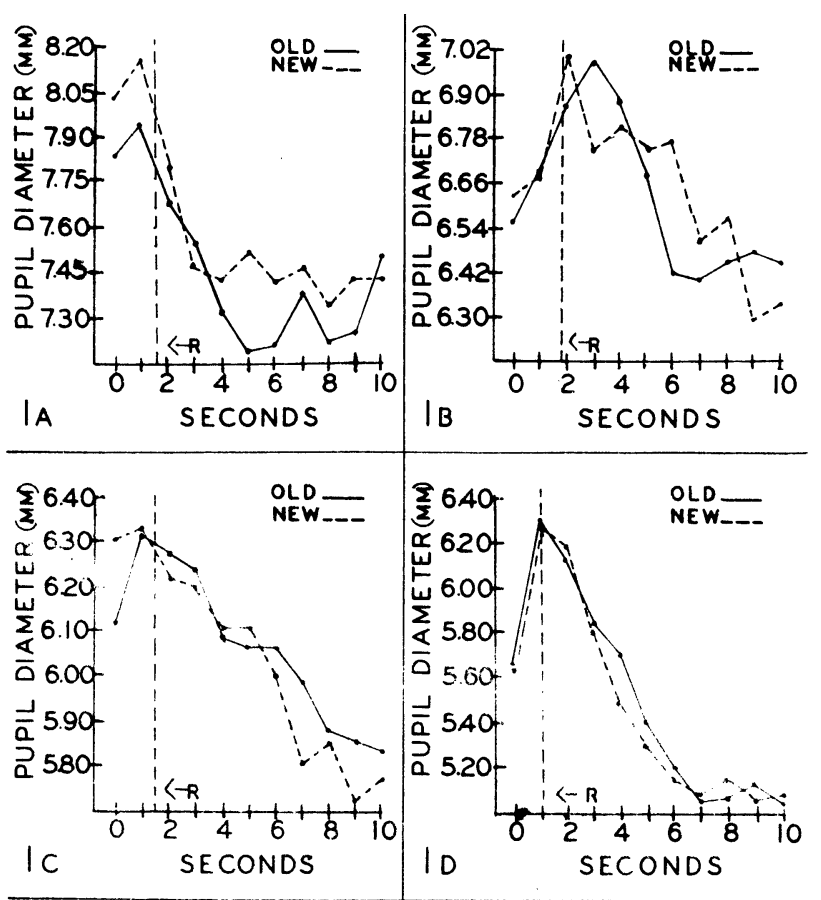

Fig. 1. Pupil diameters in millimeters with respect to reaction latency. Word items.

was calculated and is represented by a dashed vertical line.

Inspection of Fig. 1 shows that pupillary reaction to both old and new items is almost indistinguishable. The only noticeable trend is a rapid pupillary dilation to item presentation followed by progressive constriction. This observed lack of differentiation between pupillary reaction to old and new items may be due to the fact that items used consisted not only of words but also of words of high frequency level. That is, items were quite familiar whether old or new, so that little novelty was associated with presentation of new items. If this is the case, then differential pupillary reaction should be demonstrated with low-meaningfulness CCCs.

\section{EXPERIMENT II}

\section{Method}

Four undergraduate volunteers served as Ss. The procedure was identical to the previous experiment except that items were three randomly generated consonants (CCC) and 8 items instead of 10 were learned.

\section{Results}

The mean pupillary diameter with respect to old and new items is shown in Fig. 2. Differential pupillary reaction to old and new items is quite noticeable. Greater pupillary dilation seems to occur to presentation of old items than new items, and the mean pupil diameter for new items is less than that to old items throughout the 10 -sec period.

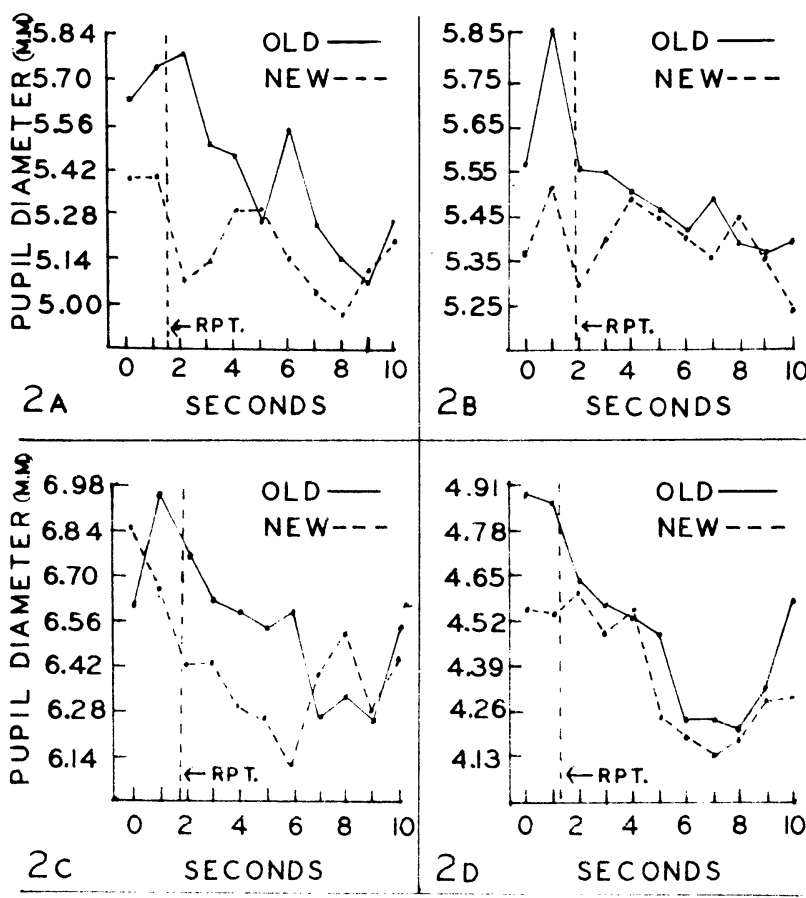

Fig. 2. Pupil diameters in millimeters with respect to reaction latency. CCC items.

Since differential pupillary reaction tends to become less toward the end of the 10-sec period, it is reasonable to infer that recognition of learned or memorized items is accompanied by much greater pupillary dilation than unlearned or nonmemorized items.

\section{DISCUSSION}

It is obvious that no noticeable orienting reflex was elicited by heteromodal novel stimuli in either experiment. If absence of the orienting reflex is interpreted as due to inhibition, then such inhibition must be viewed in light of pronounced pupillary dilation to old, and therefore familiar, stimulus items. That is, inhibition of the pupillary orienting reflex is inductively associated with the mechanism underlying pupillary dilation to old items. If the broad interpretation of Pavlov's original theory in terms of reflex directionality and task requirement is correct, then the relative absence of orienting reflex to new items constitutes a case of negative induction of the orienting reflex associated with externalization when the task requires internalization. Skolov's theory, which does not take into consideration relevance of task requirement to the direction of reflex is contradicted.

It should be noted that almost all motivationally oriented studies of pupillary reaction (e.g., Kahneman \& Beatty, 1966; Kahneman, Beatty \& Pollack, 1967; Bradshaw, 1968; Coleman \& Paivio, 1970) regard pupillary dilation as an index of mental "load," implying that pupillary reaction is a monotonic translation of motivational state. Extrapolation of this view, then predicts that the task difficulty would be monotonically related to pupillary dilation. However, if CCC items can be regarded as more difficult than word items, then this view cannot account for the fact that differential pupillary reaction to old and new items occurs only when items are relatively more difficult. It is necessary to specify with which direction mental 
load is associated, externalization or internalization. That is, in order to determine whether pupillary dilation is an attentional or motivational reaction, it is necessary to specify whether the task requirement calls for externalization or internalization and to determine if pupillary orienting reflex to stimulus novelity is inductively related to the direction of the reflex in terms of task requirement.

\section{REFERENCES}

Bradshaw, J. L. Load and pupillary changes in continuous processing tasks. British Journal of Psychology, 1968, 59, 265-271.

Coleman, F., \& Paivio, A. Pupillary dilation and mediation processes during paired-associate learning. Canadian Journal of Psy chology, 1970, 24, 261-270.

Gardner, R. M., Mo, S. S., \& Borrego, R. Inhibition of pupillary orienting reflex by novelity in conjunction with recognition memory. Bulletin of Psychonomic Society, 1974, 3, 237-238. Kahneman, D., \& Beatty, J. Pupil diameter and load on memory. Science, 1966, 154, 1583-1585.

Kahneman, D., Beatty, J., \& Pollack, I. Perceptual deficit during a mental task. Science, 1967, 157, 218-219.

Pavlov, I. P. Conditioned reflex. Oxford University Press, 1927 (Dover, 1960).

Sokolov, Ye. N. Perception and the conditioned reflex. New York: Pergamon, 1963.

Thorndike, E. L., \& Lorge, I. The teacher's word book of 30,000 words. New York: Teachers College Press, 1944.

(R eceived for publication August 5, 1974.)

\title{
A component analysis of natural language mediators obtained in paired-associate learning*
}

\author{
JERRY M. OWENS $\dagger$, PAMELA R. WERDER, and PHILIP H. MARSHALL \\ Texas Tech University, Lubbock, Texas 79409
}

\begin{abstract}
The structural relationships between paired-associate components and natural language mediators (NLMs) were analyzed by using forward and backward recall paradigms. Ss were required to give the NLM and the appropriate to-be-recalled component for pairs learned by natural language mediation. An insignificant difference was found in the ability of "stimulus" and "response" components to elicit NLMs, but a significant difference was obtained in the ability to decode the NLM into the correct response, with the forward recall procedure resulting in more efficient response recall. The results were discussed in terms of differential elaboration of stimulus and response components and in terms of Adams's (Adams \& Bray, 1970) theory of paired-associate learning.
\end{abstract}

In Adams's closed-loop theory of paired-associate (PA) verbal learning (Adams \& Bray, 1970), a natural language mediator (NLM) is considered to be any idiosyncratic verbal device which $\mathrm{S}$ uses to mediate the learning of a stimulus-response pair. Natural language mediators used during learning are detected by verbal reports. The use of NLMs has been found to increase recall in short- and long-term retention (Adams, Marshall, \& Bray, 1971a, b; Groninger, 1966; Kiess, 1968; Montague, Adams, \& Kiess, 1966) and to reduce interlist interference (A rams et al, 1971a; Adams \& Montague, 1967).

* This research was supported in part by funds from the Graduate School, Texas Tech University. The paper is sponsored by C. G. Halcomb, who takes full editorial responsibility. Requests for reprints should be sent to Philip $H$. Marshall, Department of Psychology, Texas Tech University, Lubbock, Texas 79409

+Now at the Naval Aerospace Medical Research Laboratory, Pensacola, Florida.
By Adams's (Adams \& Bray, 1970) definition, a NLM embodies both the stimulus and response terms and is considered as a response itself. The implication is that a NLM, once established, can be elicited by either the stimulus or response term. Adams allowed, however, that NLMs may have a favored direction (i.e., forward) which could suggest the possibility of greater ease of decoding the NLM for the correct response when forward rather than backward recall is required.

The purpose of this investigation was to analyze structural relationships between PA components and NLMs. To determine whether both members of the PA item are equally capaple of eliciting the common NLM, the procedures of forward and backward recall were used. In addition to response recall, Ss were required to recall the actual NLMs to determine NLM availability. They were then to recall the responses, presumably requiring decoding of the NLM, which resulted in 